$R M x A C, \mathbf{5 3}, 113-123(2021)$

(c) 2021: Instituto de Astronomía, Universidad Nacional Autónoma de México

https://doi.org/10.22201/ia.14052059p.2021.53.23

\title{
OBSERVATIONAL PROPERTIES OF TEV DETECTED GRB 180720B, GRB 190114C AND GRB 190829A
}

\author{
Rahul Gupta ${ }^{1,2}$, Shashi B. Pandey ${ }^{1}$, Alberto J. Castro-Tirado ${ }^{3,4}$, Amit Kumar $^{1,5}$, Amar Aryan ${ }^{1,2}$, \\ and S. N. Tiwari ${ }^{2}$
}

\section{RESUMEN}

Las emisiones de $\mathrm{TeV}$ de los estallidos de rayos- $\gamma$ son muy importantes para estudiar su origen y los mecanismos de radiación en detalle. Las observaciones recientes de fotones de TeV en algunos de los GRB son difíciles de explicar por el mecanismo de radiación sincrotrón tradicional. En este trabajo, presentamos los resultados de una investigación detallada de las emisiones tempranas y de la posluminiscencia de GRBs observados en el rango TeV recientemente descubiertos (GRB 180720B, GRB 190114C y GRB 190829A) por HESS y MAGIC para los que usamos datos que abarcan tanto la emisión temprana (de archivos públicos) como las posluminiscencias (por medio de observaciones del $10.4 \mathrm{~m}$ GTC y de $1.3 \mathrm{~m}$ DFOT). La espectroscopía en el rango de los rayos- $\gamma$ de alta resolución temporal de la emisión temprana para GRB 180720B y GRB 190114C muestra una correlación entre la intensidad y la energía máxima. En el caso de GRB 190829A, la energía del pico de emisión muestra una evolución de dura a suave seguida de una tendencia muy suave aunque caótica. GRB 190829A es un peculiar estallido conformado por dos episodios de emisión con un primer episodio que no sigue la correlación de Amati. Analizamos la emisión tardía de Fermi-LAT que abarca las observaciones de H.E.S.S. y MAGIC. Es probable que algunos de los fotones detectados por LAT estén asociados con estos GRB y podrían tener un mecanismo de radiación Compton inverso.

\section{ABSTRACT}

$\mathrm{TeV}$ emissions from $\gamma$-ray bursts are very important to study their origin and the radiation mechanisms in detail. Recent observations of $\mathrm{TeV}$ photons in some of the GRBs are challenging to be explained by the traditional Synchrotron radiation mechanism. In this work, we present the results of a detailed investigation of the prompt and afterglow emissions of recently discovered TeV GRBs (GRB 180720B, GRB 190114C, and GRB 190829A) based on the publicly available prompt and afterglow data including $10.4 \mathrm{~m}$ GTC and $1.3 \mathrm{~m}$ DFOT telescopes observations of the first HESS and MAGIC bursts, respectively. Time-resolved spectroscopy of prompt emission of GRB 180720B and GRB 190114C shows an intensity tracking nature of peak energy. In the case of GRB 190829A, peak energy evolution shows a hard to soft tracking trend followed by a very soft and chaotic trend. GRB 190829A is a peculiar intermediate luminous two episodic burst with first emission episode outlier to Amati correlation. We analyzed the late time Fermi-LAT emission that encapsulates the H.E.S.S. and MAGIC observations. Some of the LAT photons are likely to be associated with these GRBs and they could have an Inverse Compton radiation mechanism.

Key Words: gamma ray burst: inidividual: GRB 180720B, GRB 190114C, GRB 190829A

\section{INTRODUCTION}

Gamma-ray bursts (GRBs) are short and most violent explosions in the Universe, originated due to the death of massive stars or merging of two compact

\footnotetext{
${ }^{1}$ Aryabhatta Research Institute of Observational Sciences (ARIES), Manora Peak, Nainital-263002, India.

${ }^{2}$ Department of Physics, Deen Dayal Upadhyaya Gorakhpur University, Gorakhpur 273009, India.

${ }^{3}$ Instituto de Astrofisica de Andalucía (IAA-CSIC), Glorieta de la Astronomía s/n, E-18008, Granada, Spain.

${ }^{4}$ Unidad Asociada Departamento de Ingeniería de Sistemas y Automática, E.T.S. de Ingenieros Industriales, Universidad de Málaga, E-29071, Spain.

${ }^{5}$ School of Studies in Physics and Astrophysics, Pandit Ravishankar Shukla University, Chattisgarh 492 010, India.
}

objects such as NS-NS or NS-BH. They emit photons across the electromagnetic ( $\gamma$-rays to radio) wavebands. They are one of the most luminous (Isotropic equivalent $\gamma$-rays luminosity $\sim 10^{51}-10^{53} \mathrm{ergs} \mathrm{s}^{-1}$ ) astrophysical explosions and characterized as cosmological sources. GRBs are known to be fueled by a bipolar relativistic jet that is launched by a central engine. They have two phases of their emission the bright, short-lived and highly variable in $\mathrm{keV}$ to $\mathrm{MeV}$ channels, known as prompt emission followed by the long-lived afterglow emission. They are classified into two categories based on their duration of prompt emission. The long GRBs last for $>2 \mathrm{~s}$ while the short ones last for $\leq 2 \mathrm{~s}$. It implies a dif- 
ference between their progenitors. Long GRBs are believed to be produced due to core-collapse of massive stars whereas binary mergers of neutron stars and/or black hole are the most favored scenario for the progenitors for short GRBs (Kumar \& Zhang 2015).

Prompt emission is believed to be produced by the jet via dissipation in internal shocks or magnetic reconnection. Subsequently, ultra-relativistically ejected material interact with the surrounding medium and produce external shocks that are answerable for afterglow emission. However, the radiation process in GRBs, mainly that of the prompt emission phase is still debatable. Afterglows emission is typically well described as synchrotron radiation originated due to external forward shocks (Piran 2004). The recent detection of very high energy (VHE) sub-TeV radiation by ground-based Cerenkov telescopes (High Energy Stereoscopic System (H.E.S.S.) and Major Atmospheric Gamma Imaging Cherenkov (MAGIC)) has open new window to understand the radiation mechanisms, jet composition, jet geometry and the central engine of GRBs. These bursts are very sparse, only three GRBs (GRB 180720B, GRB 190114C, and GRB 190829A) have been reported with confirm TeV radiation up to date. Therefore each discovery of $\mathrm{TeV}$ GRB conveys enormous scientific importance to understand their mechanism in detail. Robotic telescope networks such as MASTER (Lipunov et al. 2012) and the Burst Observer and Optical Transient Exploring System ((BOOTES); Castro-Tirado $2008,2011,2016)$ can play a very significant role to quick (some of the data being contemporaneous to the prompt emission) and long follow-up observations of such GRBs.

Here, we present a detailed analysis of TeV GRB 180720B, GRB 190114C and GRB 190829A detected by the Cerenkov telescopes. Compared to the earlier two cases, GRB 190829A is the nearest one (z $\sim 0.0785)$ and exceptional intermediate luminous in the nature in the $\mathrm{TeV}$ GRBs class and thus points towards a diversity in new class of TeV GRBs. Throughout this paper, we considered the following cosmology parameters $\mathrm{H}_{0}=70 \mathrm{~km} \mathrm{~s}^{-1} \mathrm{Mpc}^{-1}, \Omega_{M}$ $=0.3$, and $\Omega_{v}=0.7$. The multiwavelength afterglow is given by a standard temporal and spectral power law, $F_{\nu} \alpha t^{-\alpha} \nu^{-\beta}$, where $\alpha$ and $\beta$ are the temporal and spectral indices, respectively. All errors are quoted at $3 \sigma$ level, unless mentioned differently.

\section{PROMPT OBSERVATIONS}

GRB 180720B triggered Swift Burst Alert Telescope (BAT) at 14:21:44 UT on 20 $0^{\text {th }}$ July 2018.
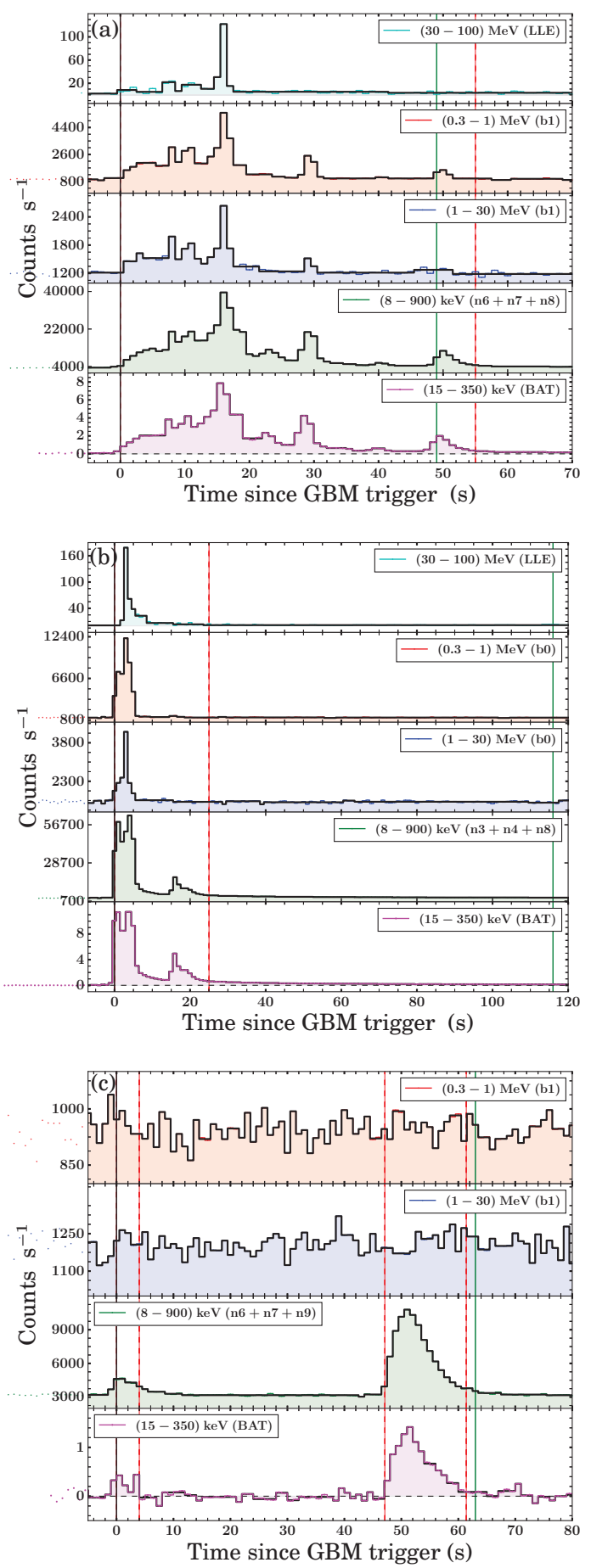

Fig. 1. Energy-resolved prompt emission light curves: The prompt emission light curves of GRB 180720B (a), GRB 190114C (b) and GRB 190829A (c) using Swift and Fermi data in different energy channel. Bayesian Blocks are over-plotted in each light curves. Solid black and green vertical lines represent the Fermi trigger time and GBM $\mathrm{T}_{90}$ duration in $50-300 \mathrm{keV}$ energy range, respectively. Red dashed vertical line shows the durations for time-averaged spectra analysis. 
Satellite slew immediately towards the source for further observations. Using the Swift BAT data, the best-on-ground location was found at RA, Dec = $0.530,-2.933$ (J2000) having an uncertainty radius of $3^{\prime}$. The BAT light curve consists of multiple-peak structure with a total duration of $\sim 150 \mathrm{~s}$ (Siegel et al. 2018). GRB was detected by the Fermi GammaRay Burst Monitor (GBM) at 14:21:39.65 UT and Large Area Telescope (LAT) at 14:21:44.55 UT. The most effective GBM on-ground position of the burst was consistent with the Swift location. GBM light curve shows multiple very bright peaks with numerous overlapping pulses with $\mathrm{T}_{90}$ of the GRB was about $49 \mathrm{~s}$ in GBM 50-300 keV energy channels (Roberts \& Meegan 2018; Bissaldi \& Racusin 2018). Prompt emission was also detected by MAXI/GSC at 14:28:15 UT (Negoro et al. 2018), CALET Gamma-ray Burst Monitor (CGBM) at 14:21:40.948 UT (Cherry et al. 2018) and Konus-Wind satellite at 14:21:45.261 UT (Frederiks et al. 2018).

GRB 190114C triggered Swift BAT at 20:57:03 UT on $14^{\text {th }}$ January 2019 . The best-on-ground location was found using BAT data at RA, Dec = $54.510,-26.939$ (J2000) having an uncertainty radius of $3^{\prime}$. The BAT light curve consists of a very bright multiple-peak structure with a total duration of $\sim$ $25 \mathrm{~s}$ (Gropp et al. 2019). GRB was detected by the Fermi GBM and LAT simultaneously at 20:57:02.63 UT. GBM light curve shows a very bright multiple peak pulses with $\mathrm{T}_{90}$ of the GRB was about $116 \mathrm{~s}$ in GBM 50-300 keV energy channels (Hamburg et al. 2019; Kocevski et al. 2019). Prompt emission was also detected by AGILE/MCAL at 20:57:02.26 UT (Ursi et al. 2019), Insight-HXMT/HE at 20:57:02.63 UT (Xiao et al. 2019) and Konus-Wind satellite at 20:57:02.341 UT (Frederiks et al. 2019).

GRB 190829A triggered Fermi GBM at 19:55:53 UT $\left(\mathrm{T}_{0}\right)$ on $29^{\text {th }}$ August 2019 (Lesage et al. 2019) and Swift BAT at 19:56:44.60 UT (Lien et al. 2019). The best on-ground location was found using BAT data at RA, Dec $=44.540,-8.968$ (J2000) having an uncertainty radius of $3^{\prime}$. The BAT light curve consists of smaller peak followed by the main peak (Dichiara et al. 2019). The best GBM on-ground position of the burst was consistent with the Swift location. Prompt emission was also detected by $A G$ ILE at 19:55:53 UT (Pittori et al. 2019) and KonusWind in the waiting mode (Tsvetkova et al. 2019). Fermi and Swift both detected two episodes during the prompt emission, the first episode starting from $\mathrm{T}_{0}$ to $\mathrm{T}_{0}+4 \mathrm{~s}$ followed by a softer episode from $\mathrm{T}_{0}$ $+47.1 \mathrm{~s}$ to $\mathrm{T}_{0}+61.4 \mathrm{~s}$. The spectrum of the first episode using the Fermi data is best described by

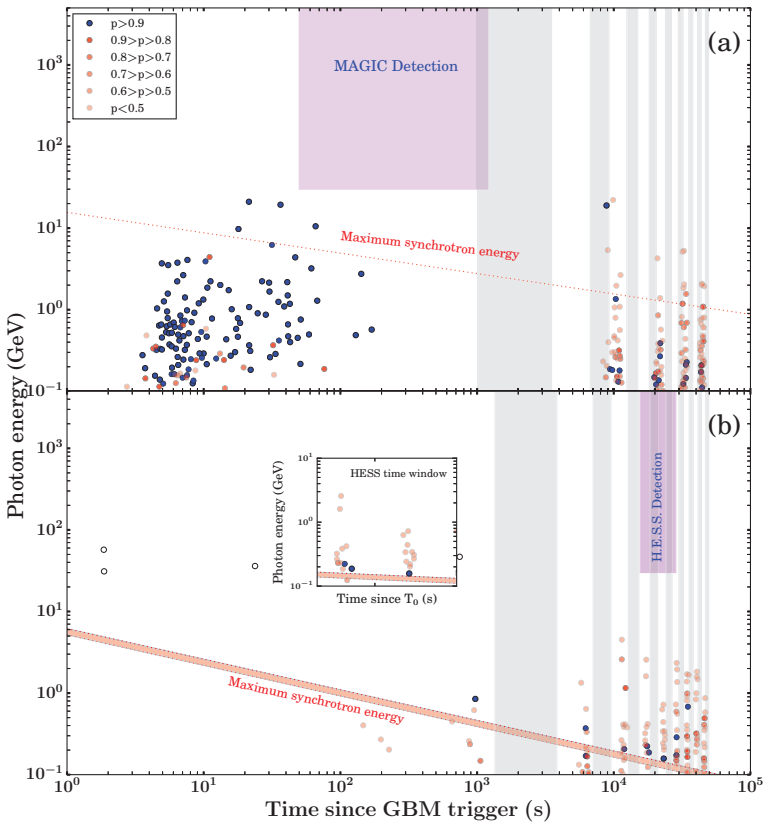

Fig. 2. High energy LAT photons with energies $>100$ $\mathrm{MeV}$ and their probabilities of being linked with GRB 190114C and GRB 190829A in subplot (a), and (b), respectively. The shaded grey bars represent the regions with zenith angle $>100^{\circ}$. The red shaded lines or region shows the maximum photon energies originated for a synchrotron external forward-shock model. The small inset in subplot (b) represent the LAT high energy emission during the H.E.S.S. observations.

a power-law with an exponential high-energy cutoff function having an index of $-1.41 \pm 0.08$, and a cutoff energy corresponding to a peak energy, $\mathrm{E}_{p}=130$ $\pm 20 \mathrm{keV}$. Where as the second episode is best fit by a Band function (Band et al. 1993) with $\mathrm{E}_{p}=11 \pm$ $1 \mathrm{keV}, \alpha=-0.92 \pm 0.62$ and $\beta=-2.51 \pm 0.01$. The observed fluence is $1.27 \pm 0.02 \times 10^{-5} \mathrm{erg} \mathrm{cm}^{-2}$ in the $10-1000 \mathrm{keV}$ band (Lesage et al. 2019).

\section{MULTIWAVELENGTH DATA ANALYSIS}

\subsection{Fermi Large Area Telescope}

We downloaded the Fermi LAT data using Fermi Science Tools for a time interval of $50000 \mathrm{~s}$ after Fermi trigger time for each of the TeV GRBs. We analyzed the LAT data using software gtburst. To carry out unbinned likelihood investigation, we selected a region of interest (ROI) of $12^{\circ}$ around the enhanced Swift XRT position. We purified the high energy LAT emission by putting a cut to photons with energies in the range $100 \mathrm{MeV}-300 \mathrm{GeV}$. Further, an angle cut of $100^{\circ}$ between the GRB location and zenith of the satellite was applied in order to reduce the contamination of photons arriving from the Earth 
limb based on the navigation plot for the respective burst. For the full-time intervals, we employed the P8R3_SOURCE_V2 response, which is appropriate for longer durations $\left(\sim 10^{3} \mathrm{~s}\right)$ and for short temporal bins, we used P8R2_TRANSIENT020E_V6 response, which is appropriate for small durations. The probability of these high energy photons to be associated with the source is calculated with the help of gtsrcprob tool.

For GRB $180720 B$, the burst location was at $50^{\circ}$ from the LAT boresight angle at the time of the GBM trigger. The best LAT on-ground position of the burst was at RA, DEC $=0.58,-2.95$ (J2000) with a $90 \%$ uncertainty radius of 0.11 degree. LAT detected few photons with energy above $1 \mathrm{GeV}$. The highest-energy photon with energy $\sim 5 \mathrm{GeV}$ was detected $137 \mathrm{~s}$ after the GBM trigger (Bissaldi \& Racusin 2018). For GRB 190114C, the source position was $68^{\circ}$ from LAT boresight angle at the time of the GBM trigger. The best LAT on-ground position of the burst was at RA, DEC $=54.57,-26.99$ (J2000) with a $90 \%$ uncertainty radius of 0.05 degree. The highest-energy photon with energy $\sim 22.9 \mathrm{GeV}$ was detected $15 \mathrm{~s}$ after the GBM trigger (Kocevski et al. 2019). In the case of GRB 190829A, the GRB position was $33^{\circ}$ from the LAT boresight angle at the time of the GBM trigger. No high-energy gamma-ray emission was detected by the LAT in the initial interval or any subsequent intervals (Piron et al. 2019). Our late time analysis covering the H.E.S.S. observation window shows the detection of three high energy photons with probability $>90 \%$ of their coalition with the GRB, Figure 2 (c). We consider an emission efficiency in the range $\eta=0.1-0.4$ for GRB 190829A.

To probe the emission mechanisms of these high energy LAT photons, we measured the maximum photon energy emitted by the synchrotron process during the deceleration phase (Fraija et al. 2019). The red dashed lines in Figure 2 (a), and red-shaded region in Figure 2 (b) show the maximum photon energies released by the synchrotron radiation due to external forward shock. The Fermi LAT high energy photons placed below these red lines/regions are consistent with the prediction of the external forward shock model. However, some of the LAT photons placed above these lines/region which might be produced due to the synchrotron self-Compton radiation mechanism (MAGIC Collaboration et al. 2019; Abdalla et al. 2019; Chand et al. 2020).

\subsection{Fermi Gamma-ray Burst Monitor}

We obtained the Fermi GBM data from GBM GRBs catalog ${ }^{6}$ using gtburst software. We studied the temporal and spectral prompt emission properties of TeV GRBs using at least three bright NaI and one BGO detectors. To perform the spectral analysis, we reduced the time-tagged event (TTE) mode data using Fermi Science Tools software gtburst $^{7}$ as they have high time precision in all the 128 energy channels. We performed the modeling of time-averaged spectra using X-Ray Spectral Fitting Package XSPEC (Arnaud 1996). We began joint GBM and LAT (if available) data modeling with the Band function and used the various other models based upon model fit, residuals of the data, and their parameters. The statistics pgstat is used for optimization and testing the various models. The functional form of the Band model used to fit the photon spectrum is traditional $\mathrm{grbm}$ model available in $\mathrm{XSPEC}^{8}$ (Band et al. 1993). In the model $\alpha$, $\beta$ and $\mathrm{E}_{0}$ corresponds to low energy spectral index, high energy spectral index and break energy, respectively. The spectral peak energy $\left(\mathrm{E}_{p}\right)$ is equal to $(2+$ $\alpha) \mathrm{E}_{0}$.

For the temporal and spectral analysis of GRB 180720B, we used three bright NaI detectors (n6, n7, and n8) with source observing angles (n8: $46^{\circ}$, $\left.\mathrm{n} 7: 10^{\circ}, \mathrm{n} 6: 29^{\circ}\right)$. We selected one BGO (b1) detector out of two BGO detectors, as it is closer to the direction of the GRB. The angle constraints are to avoid the systematics arising due to uncertainty in the response at larger angles. Fermi and Swift multi-channel light curve is shown in Figure 1 (a). The light curve consists of several merging emission episodes. In the case of MAGIC burst, we used three NaI detectors (n3, n4, and n8) and one BGO (b0) detector which has significant count rates and their GRB observing angles( n3: $41^{\circ}, \mathrm{n} 4: 37^{\circ}, \mathrm{n} 8: 41^{\circ}$, b0: $88^{\circ}$ ) for the temporal analysis. We included one more $\mathrm{NaI}(\mathrm{n} 7)$ and other BGO (b1) detectors for spectral analysis to constrain the parameters. Fermi and Swift multi-channel light curve is presented in Figure 1 (b). The light curve consists of two emission episodes. The second episode is softer in nature. For GRB 190829A, we used three NaI detectors (n6, $\mathrm{n} 7$ and n9) and one BGO (b1) detector with their source observing angles (n9: 49 $, \mathrm{n} 7: 14^{\circ}, \mathrm{n} 6$ : $\left.12^{\circ}\right)$. We did not use any BGO detector in the spec-

\footnotetext{
${ }^{6}$ https://heasarc.gsfc.nasa.gov/W3Browse/fermi/ fermigbrst.html

${ }^{7}$ https://fermi.gsfc.nasa.gov/ssc/data/analysis/ scitools/gtburst.html

${ }^{8}$ https://heasarc.gsfc.nasa.gov/xanadu/xspec/ manual/node179.html
} 
tral analysis as they do not show any strong emission feature. Fermi multi-channel light curve for these bright detectors is shown in Figure 1 (c). The light curve consists of two episodes separated by a quiescent time interval around $40 \mathrm{~s}$. The second episode is very soft in nature.

The time-averaged spectrum of GRB 180720B is best modelled with band function, Figure 3 (a). For GRB 190114C, the time-averaged spectrum shows a high energy cutoff when modelled with band function along with cutoffpow model, Figure 3 (c). The first emission episode of GRB 190829A is modelled with cutoffpow model, Figure 3 (d) and the second emission episode follows the typical band function with a very soft peak energy, Figure 3 (e). All the spectral parameters for TeV GRBs are listed in Table 1.

\subsection{Amati correlation and fluence distribution}

We placed the TeV GRBs in the Amati correlation plane of long GRBs along with the data points for long GRBs used in (Goldstein et al. 2017). GRB 180720B and GRB 190114C well follow the Amati correlation. But the first hard emission episode of GRB 190829A is an outlier to the Amati correlation of long GRBs (Chand et al. 2020). The second episode lies at the lower ends of the correlation (see the left panel of Figure 4). We plotted the fluence distribution of these sources along with rest-frame $T_{90}$ duration in right panel of Figure 4 . This distribution confirmed that GRB 180720B and GRB 190114C have nearly similar fluence. But GRB 190829A shows less fluence. It could be the case that GRB 190829A was intrinsically faint.

\subsection{Time Resolved Spectroscopy}

Time-resolved spectroscopy is an important tool to understand the emission mechanism and spectral parameter evolution of GRBs in detail. Peak energy $E_{p}$ can have three types of the evolution patterns, (i) 'hard-to-soft' trend, peak energy will be decreasing continuously (Bhat et al. 1994; Band 1997); (ii) flux-tracking trend, peak energy will be increasing/decreasing as the flux is increasing/decreasing (Golenetskii et al. 1983; Ryde \& Svensson 1999); (iii) 'soft-to-hard' trend or disordered trend (Laros et al. 1985; Kargatis et al. 1994). The evolution of low energy spectral index $(\alpha)$ does not show any strong usual pattern in spite of the fact that it evolves with time in place of remaining constant. We have taken the time-resolved spectroscopy results for GRB 180720B, GRB 190114C and GRB 190829A from (Duan \& Wang 2019), (Chand et al. 2019) and (Chand et al. 2020), respectively.
Temporal evolution of peak energy of GRB 180720B and GRB 190114C follow the flux tracking pattern. In the case of GRB 190829A, the evolution of the first episode follows the hard to soft (hst) pattern and the second episode shows disordered peak energy evolution. In case of GRB 180720B, low energy spectral index $(\alpha)$ do not run over the synchrotron limits (from -1.5 to -0.67). But in the case of GRB 190114C and GRB 190829A, it overshoots the synchrotron limits which could be due to the presence of some other component such as the black body in their prompt emission phase other than traditional non-thermal synchrotron radiation.

\subsection{Swift Burst Alert Telescope}

We extracted the Swift BAT light curves and time-averaged spectra using the standard analysis technique $^{9}$. We modeled the spectra using XSPEC package. For GRB 180720B, the time-averaged BAT spectrum from $\mathrm{T}_{0}-20.0 \mathrm{~s}$ to $\mathrm{T}_{0}+961.1 \mathrm{~s}$ is best fitted with a simple power-law model with power law index alpha $=1.36 \pm 0.03$. The BAT fluence observed in the $15-150 \mathrm{keV}$ band is $(8.6 \pm 0.1) \times$ $10^{-5} \mathrm{erg} \mathrm{cm}^{-2}$ (Barthelmy et al. 2018). In the case of GRB 190114C, the time-averaged BAT spectrum from $\mathrm{T}_{0}-6.604 \mathrm{~s}$ to $\mathrm{T}_{0}+703.192 \mathrm{~s}$ is best fitted with a simple power-law model with power law index alpha $=1.43 \pm 0.02$. The BAT fluence observed in the $15-150 \mathrm{keV}$ band is $(8.3 \pm 0.1) \times 10^{-5} \mathrm{erg} \mathrm{cm}^{-2}$ (Krimm et al. 2019). For the most recent TeV detected GRB 190829A, the time-averaged BAT spectrum from $\mathrm{T}_{0}-51.9 \mathrm{~s}$ to $\mathrm{T}_{0}+7.2 \mathrm{~s}$ is best fitted with a simple power-law model with $\Gamma=2.56 \pm 0.21$. The BAT fluence observed in the $15-150 \mathrm{keV}$ band is $(6.4 \pm 0.7) \times 10^{-6} \mathrm{erg} \mathrm{cm}^{-2}$ (Lien et al. 2019). The light curves emission episodes extracted using BAT observations in the full energy channel are consistent with Fermi light curve for each GRBs (see Figure 1).

\subsection{Swift X-ray Telescope}

The Swift X-ray telescope (XRT) mainly observe the X-ray counterparts of GRBs in $0.3-10 \mathrm{keV}$ energy range. We obtained the XRT light curve and the spectrum from the Swift online repository ${ }^{10}$. We analyzed the XRT spectrum in its full energy (0.3 $10 \mathrm{keV}$ ) range. We considered absorption components along with the source spectral model to constrain the intrinsic hydrogen column density. For the absorption components, we choose a fixed Galactic column density from the (Willingale et al. 2013) and

\footnotetext{
${ }^{9}$ https://www.swift.ac.uk/analysis/bat/index.php

10 https://www.swift.ac.uk/xrt_curves/ https://www.swift.ac.uk/xrt_spectra/
} 

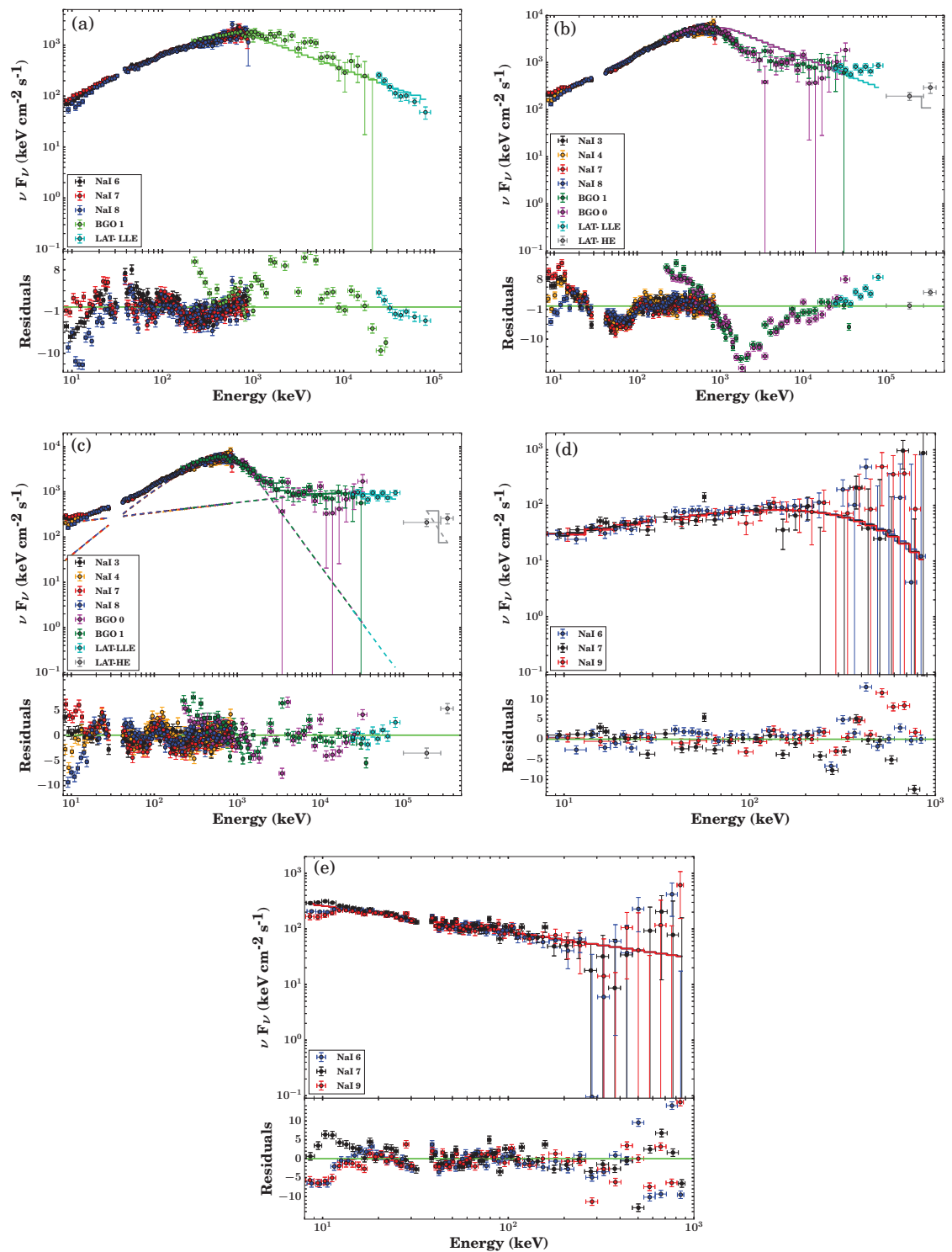

Fig. 3. The time-integrated unfolded spectrum of TeV GRBs : (a) GRB 180720B (0.0 - 55.0 s) fitted with the Band model, (b) GRB 190114C (0.0 - $25.0 \mathrm{~s})$ fitted with the Band model (c) similar as (b) along with additional power-law model having high energy cutoff (d) GRB 190829A (0.0 - $4.0 \mathrm{~s})$ fitted with the cutoff power-law model. (e) second emission episode of GRB 190829A (47.1- $61.4 \mathrm{~s}$ ) fitted with the power-law model. Bottom panels show the residuals in the respective plots.

a free intrinsic column density corresponding to the host redshift. We searched for additional thermal and other possible components in the spectra. All the parameters along with various model have been presented in Table 2.

For the first H.E.S.S. detected GRB, XRT started observing the BAT field $86.5 \mathrm{~s}$ after the BAT trigger. A new bright X-ray object was detected at RA, DEC $=0.5279,-2.9170$ (J2000), consistent with the Swift BAT error circle with a $90 \%$ uncertainty ra- dius of 5" (Siegel et al. 2018). In the case of MAGIC GRB, XRT started observing the BAT field $64 \mathrm{~s}$ after the BAT trigger. A new bright X-ray object was detected at RA, DEC $=54.5068,-26.9467$ (J2000), consistent within the Swift BAT error circle with a $90 \%$ uncertainty radius of $5 "$ (Gropp et al. 2019). For GRB 190829A, XRT started observing the BAT localization region to search for an X-ray counterpart of the burst at 19:58:21.9 UT, 97.3 s after the BAT trigger, beginning with window timing (WT) mode. 

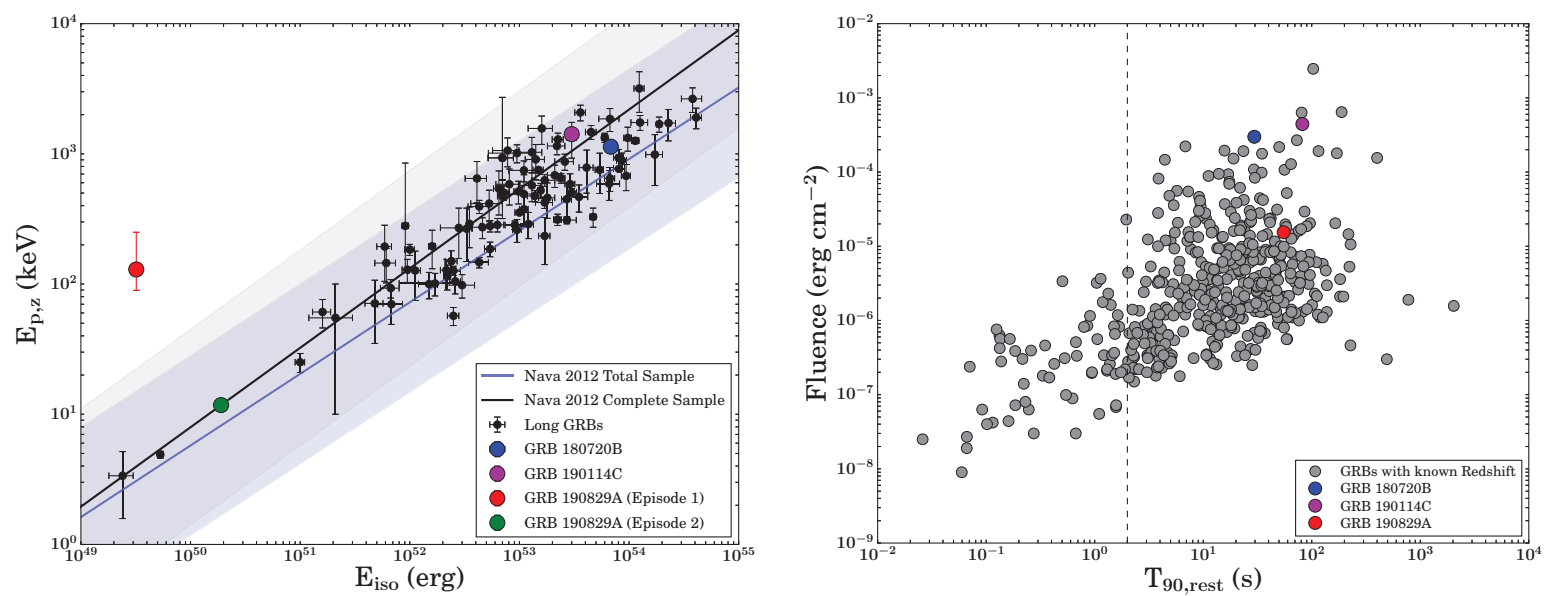

Fig. 4. The left panel shows Amati Correlations for TeV GRBs: The TeV GRBs along with the data points for long GRBs used in (Goldstein et al. 2017) in Amati correlation plane. The shaded region represents the $3 \sigma$ scatter of the correlations (Nava et al. 2012). The first episode of GRB 190829A is an outlier to the Amati correlation. The right panel shows rest frame $\mathrm{T}_{90}$ vs fluence distribution for GRBs with known redshift along with TeV GRBs.

TABLE 1

TIME-AVERAGED SPECTRAL ANALYSIS RESULT USING FERMI GBM AND LAT DATA OF TEV GRBS

\begin{tabular}{|c|c|c|c|c|c|c|c|}
\hline GRB & Model & $\alpha$ & $\beta$ & $E_{p}(\mathrm{keV})$ & $E_{c}(\mathrm{keV})$ & $\Gamma$ & pgstat/dof/BIC \\
\hline GRB 180720B & Band & $-1.11_{-0.01}^{+0.01}$ & $-2.65_{-0.03}^{+0.03}$ & $745.26_{-24.79}^{+25.47}$ & & & $1890 / 468 / 1921$ \\
\hline GRB $190114 \mathrm{C}$ & $\begin{array}{c}\text { Band } \\
\text { Band+Cutoffpow }\end{array}$ & $\begin{array}{c}-1.021_{-0.004}^{+0.004} \\
-0.48_{-0.02}^{+0.02} \\
\end{array}$ & $\begin{array}{l}-2.70_{-0.02}^{+0.02} \\
-4.50_{-1.24}^{+0.51} \\
\end{array}$ & $\begin{array}{c}926.46_{-16.99}^{+17.11} \\
428.398_{-10.54}^{+10.84} \\
\end{array}$ & $9.83_{-1.34}^{+1.73} \times 10^{4}$ & $1.78_{-0.01}^{+0.01}$ & $\begin{array}{l}5059 / 699 / 5091 \\
1864 / 696 / 1917 \\
\end{array}$ \\
\hline $\begin{array}{l}\text { GRB } 190829 \mathrm{~A} \\
\text { Episode } 1 \\
\text { Episode } 2\end{array}$ & $\begin{array}{l}\text { Cutoffpow } \\
\text { Band } \\
\text { Power-law }\end{array}$ & $-0.92_{-0.62}^{+0.62}$ & $-2.51_{-0.01}^{+0.01}$ & $11_{-1}^{+1}$ & $226.75_{-84.12}^{+217.29}$ & $\begin{array}{l}1.39_{-2.71}^{+3.82} \\
2.48_{-0.01}^{+0.01}\end{array}$ & $\begin{array}{c}356 / 344 / 374 \\
\text { GCN 25575 } \\
820 / 459 / 839\end{array}$ \\
\hline
\end{tabular}

A bright and uncatalogued X-ray afterglow candidate was detected at RA, DEC $=44.5440,-8.9579$ (J2000), consistent within the Swift BAT error circle with a $90 \%$ uncertainty radius of $2 "$ in the initial WT exposure (Dichiara et al. 2019). It switched to photon counting $(\mathrm{PC})$ mode $\sim 243 \mathrm{~s}$ after the BAT trigger time. The $\mathrm{X}$-ray afterglow was monitored until $\sim 10^{7}$ s post-trigger. We modelled the XRT light curve using smoothly broken power-law or power-law only.

\subsection{Swift UVOT and Optical data}

We collected Swift Ultra-Violet and Optical telescope (UVOT) data from the online Swift archive page $^{11}$. For the analysis of UVOT data, we use HEASOFT software version 6.25 with the latest calibration database. We performed the reduction of the UVOT data using standard uvotproduct pipeline. A source circular region of 5 " and a background region

\footnotetext{
${ }^{11}$ https://www.swift.ac.uk/swift_portal/
}

of 25 " aperture radius was extracted for the analysis. As the source becomes faint at later epoch, all the late time sky images were stacked after their alignment to perform the photometry of the source using uvotsource pipeline (if visible after stacking or their upper limits). We summed the extensions within a sky image using uvotimsum. For summing the sky images from different observations, we merged the images first, using fappend. Results are shown in Figure 6 . The optical follow-up of TeV GRBs started soon after the Fermi and Swift trigger. We obtained optical and NIR afterglows data from the Gammaray coordinates network ${ }^{12}$.

For GRB 180720B, no UVOT data were available during initial observation (Siegel et al. 2018). For GRB 190114C, the Swift UVOT detector started observing the field to search for the optical/UV coun-

\footnotetext{
${ }^{12}$ https://gcn.gsfc.nasa.gov/other/180720B.gcn3, https://gcn.gsfc.nasa.gov/other/190114C.gcn3, https: //gcn.gsfc.nasa.gov/other/190829A.gcn3
} 
TABLE 2

THE X-RAY SPECTRAL ANALYSIS RESULTS OF TEV GRBS

\begin{tabular}{|c|c|c|c|c|c|c|}
\hline GRB & Mode & $\begin{array}{c}\text { Time interval } \\
\text { (s since BAT trigger) }\end{array}$ & $\begin{array}{c}\text { Net exposure } \\
(\mathrm{ks})\end{array}$ & $\begin{array}{c}\mathrm{N}_{\mathrm{H}}(\mathrm{z}) \\
\left(10^{21} \mathrm{~cm}^{-2}\right)\end{array}$ & $\Gamma$ & $\begin{array}{c}\text { Flux } \\
\left(10^{-10} \mathrm{erg} \mathrm{cm}^{-2} \mathrm{~s}^{-1}\right)\end{array}$ \\
\hline GRB 180720B & $\begin{array}{l}\text { WT } \\
\text { PC }\end{array}$ & $\begin{array}{l}90-19397 \\
90-19397\end{array}$ & $\begin{array}{l}2.2 \\
3.9\end{array}$ & $\begin{array}{l}3.71_{-0.11}^{+0.11} \\
3.40_{-0.50}^{+0.60} \\
\end{array}$ & $\begin{array}{l}1.70_{-0.01}^{+0.01} \\
1.79_{-0.06}^{+0.06} \\
\end{array}$ & $\begin{array}{c}33.33_{-0.22}^{+0.22} \\
1.89_{-0.07}^{+0.07} \\
\end{array}$ \\
\hline GRB $190114 C$ & $\begin{array}{l}\text { WT } \\
\text { PC }\end{array}$ & $\begin{array}{l}67-57216 \\
67-57216\end{array}$ & $\begin{array}{c}0.558 \\
18.8\end{array}$ & $\begin{array}{l}88.5_{-0.22}^{+0.22} \\
80.0_{-5.0}^{+6.0}\end{array}$ & $\begin{array}{l}1.71_{-0.04}^{+0.04} \\
1.93_{-0.10}^{+0.10}\end{array}$ & $\begin{array}{l}95.60_{-1.20}^{+1.20} \\
0.33_{-0.01}^{+0.01}\end{array}$ \\
\hline GRB 190829A & $\begin{array}{l}\text { WT } \\
\text { PC }\end{array}$ & $\begin{array}{l}103-46017 \\
103-46017 \\
\end{array}$ & $\begin{array}{c}0.128 \\
10.8\end{array}$ & $\begin{array}{c}9.2_{-1.8}^{+2.1} \\
13.86_{-0.97}^{+1.02} \\
\end{array}$ & $\begin{array}{l}2.21_{-0.18}^{+0.20} \\
2.19_{-0.08}^{+0.08} \\
\end{array}$ & $\begin{array}{l}3.44_{-0.28}^{+0.30} \\
0.80_{-0.03}^{+0.03} \\
\end{array}$ \\
\hline
\end{tabular}

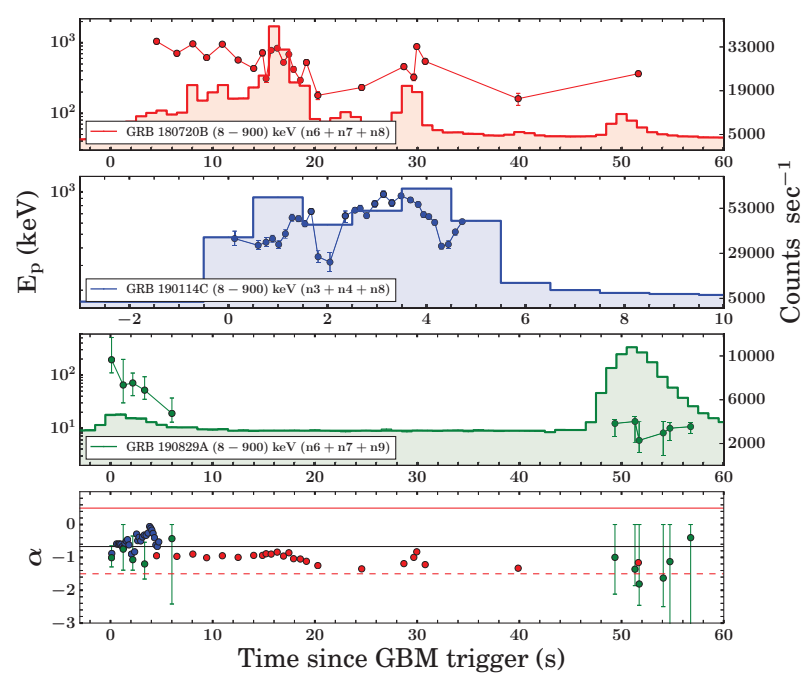

Fig. 5. Time-resolved spectroscopy of TeV GRBs. Top three panels shows the peak energy evolution. The bottom panel shows the low energy spectral index $(\alpha)$ evolution. The evolution of $\alpha$ does not show any strong usual pattern in spite of the fact that it evolves with time in place of remaining constant. Red dashed and the black solid line represents the synchrotron fast cooling spectral index of $-3 / 2$ and the line of death of synchrotron emission i.e., $-2 / 3$, respectively.

terpart of GRB at $73 \mathrm{~s}$ after the BAT trigger starting with $150 \mathrm{~s}$ exposure in the white filter (Gropp et al. 2019). A bright and rapidly fading afterglow candidate was detected at RA, DEC $=54.50484$, 26.94637 (J2000) consistent with the Swift/XRT position with a $90 \%$ uncertainty radius of $0.42^{\prime \prime}$ in the initial UVOT exposure (Siegel \& Gropp 2019). Swift UVOT light curve has been presented in Figure 6 (b). For GRB 190829A, the Swift UVOT payload took a finding chart of initial exposure of $150 \mathrm{~s}$ with the white filter starting $106 \mathrm{~s}$ after the BAT trigger. A credible rising optical afterglow candidate has been found in the initial data products. UVOT light curve has been shown in Figure 6 (c). The UVOT light curve shows the onset of the afterglow followed by a power-law decay. Onset time from UVOT light curve shows achromatic nature in different filters. Onset peak time in UVOT also consistent with the peak time of X-ray afterglow.

We carried out optical observations with the $10.4 \mathrm{~m}$ Gran Telescopio Canarias (GTC). For GRB 180720B, observations at two epochs (8 \& 18 Aug 2018) were obtained and GTC provided the redshift for both GRB 190114C $(\mathrm{z}=0.4245 \pm 0.0005$, (Castro-Tirado et al. 2019)) and 190829A ( $\mathrm{z}=$ $0.0785 \pm 0.005$, (Valeev et al. 2019)). We also observed the optical afterglow of GRB 190114C using ARIES 1.3m Devasthal Fast Optical Telescope (DFOT). We analyzed data using the Image Reduction and Analysis Facility (IRAF) and Dominion Astrophysical Observatory photometry (DAOPHOT II). Photometry results are listed in Table 3.

\subsection{Low frequency data}

Radio afterglow data is useful to constrain the self-absorption frequency from the spectral energy distribution. For GRB 180720B, Sfaradi et al. 2018 reported a low-frequency counterpart with the Arcminute Microkelvin Imager Large Array at 15.5 GHz. Afterglow was also detected with GMRT at $1.4 \mathrm{GHz}$ at the optical position (Chandra et al. 2018). For GRB 190114C, Alexander et al. 2019 detected a radio source with a flux density of $3.1 \mathrm{mJy}$ at $33.5 \mathrm{GHz}$ using VLA. A fading $\mathrm{mm}$ afterglow was observed at $90 \mathrm{GHz}$ using ALMA telescope (Laskar et al. 2019). Counterpart at $36.8 \mathrm{GHz}$ band with RT-22 telescope was detected by (Volvach et al. 2019). Other low-frequency telescopes also observed the counterpart of the MAGIC GRB ${ }^{13}$. For GRB 190829A, the radio counterpart of the burst was observed with upgraded Giant Metrewave Radio Telescope (uGMRT), starting at 02.11 UT in September 2019 in the radio frequency band (1050 $1450 \mathrm{MHz}$ ) at the position of the optical counterpart of GRB 190829A (Chandra et al. 2019). cm-band af-

\footnotetext{
${ }^{13}$ https://gcn.gsfc.nasa.gov/other/190114C.gcn3
} 

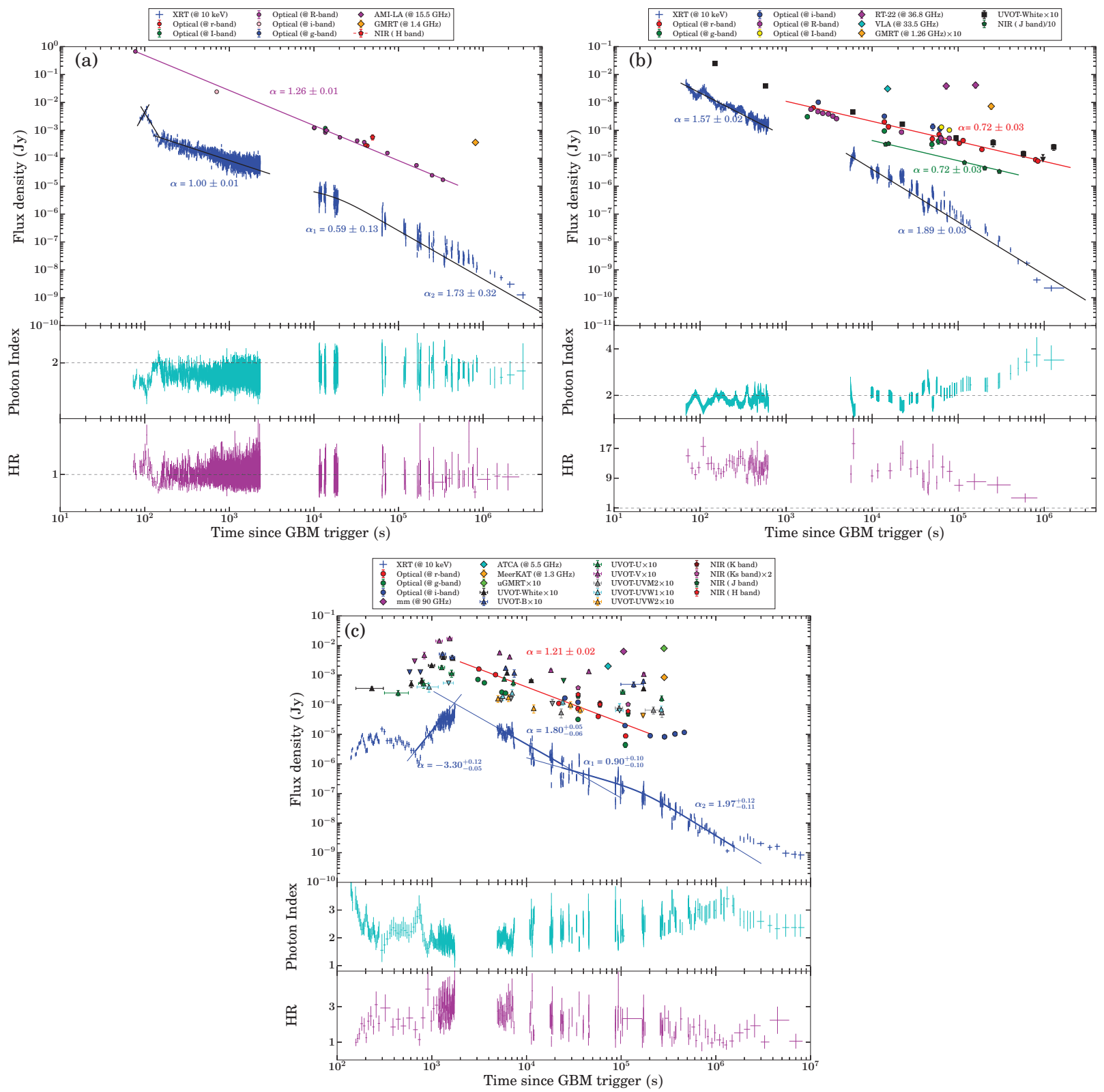

Fig. 6. Multi-band light curves of the afterglow of GRB 180720B (a), GRB 190114C (b) and GRB 190829A (c) (Chand et al. 2020) from X-ray to the radio band. The evolution of $\Gamma$ and hardness ratio are shown in the middle and bottom panel of respective plots.

terglow was detected using Australia Telescope Compact Array (ATCA), 20.2 hours after the GRB having a preliminary flux density of $\sim 2 \mathrm{mJy}$ at $5.5 \mathrm{GHz}$ (Laskar et al. 2019). mm counterpart was discovered by (de Ugarte Postigo et al. 2019) using NOEMA at $90 \mathrm{GHz}$.

\subsection{Very high energy data}

Very high energy (VHE) photons from these GRBs were detected by Cherenkov telescope arrays,
HESS and MAGIC. For GRB 180720B, the HESS collaboration presented the detection of late time VHE photons in the energy channel from 100- 400 GeV during the CTA Science Symposium 2019. In the case of GRB 190114C, the MAGIC collaboration reported VHE emission with the significance of $>20$ sigma in the first 20 min (Mirzoyan et al. 2019). GRB 190829A was second HESS burst in which late time VHE emission has been seen with $>5$ sigma gamma-ray excess in the direction of 
TABLE 3

LOG OF GRB 190114C AFTERGLOW OBSERVATIONS USING 1.3M ARIES DFOT TELESCOPE

\begin{tabular}{ccccc}
\hline UTC & $\begin{array}{c}\text { T-T } \\
\text { (days) }\end{array}$ & Filter & Mag & Telescope \\
\hline $2019-01-15$ UT 14:19:21 & 0.72375 & $\mathrm{R}$ & 19.39 & 0.06 \\
$2019-01-15$ UT 14:26:25 & 0.72875 & $\mathrm{R}$ & $1.3 \mathrm{~m} \mathrm{DFOT}$ & 0.07 \\
$2019-01-15$ UT 14:41:23 & 0.73917 & $\mathrm{I}$ & $1.3 \mathrm{~m} \mathrm{DFOT}$ & 0.04 \\
\hline
\end{tabular}

GRB 190829A based on a preliminary analysis (de Naurois \& H. E. S. S. Collaboration 2019).

\section{SUMMARY AND DISCUSSION}

We highlighted the observational prompt and afterglow properties of GRB 180720B, GRB 190114C and GRB 190829A using multiwavelength publicly available data. Multi-channel light curves of prompt emission phase of these bursts consist features of episodic activities and all of these GRBs belong to the long GRB class. Also, the last episode of all these GRBs is softer in nature. In the case of GRB 190829A, both the emission episodes are separated by a quiescent phase of $\sim 40 \mathrm{~s}$.

We analyzed the high energy photon light curves using Fermi LAT data of these GRBs and calculated the maximum photon energy emitted by the synchrotron process. The energy of some of the LAT photons lies above the maximum synchrotron energy line indicating that these photons may be produced from some other mechanism, most probably the self synchrotron compton (SSC) radiation mechanism (Joshi \& Razzaque 2019).

We found that Amati correlation is satisfied for GRB 180720B and GRB 190114C. But the episodic analysis of GRB 190829A shows that the first episode of this burst is the outlier to Amati correlation of long GRBs and the second episode lies the lower edge of the correlation. Figure 4 shows the fluence vs rest frame $\mathrm{T}_{90}$ distribution of these GRBs along with GRBs known redshift. It shows that GRB 180720B and GRB 190114C have typically similar fluence. But the GRB 190829A was fainter in comparison to the other two TeV GRBs. In fact, GRB 190829A is a intermediate luminous GRB and could have shock breakout origin (Chand et al. 2020).

Time-resolved spectral analysis shows that peak energy evolution for GRB 180720B and GRB $190114 \mathrm{C}$ have flux tracking trends. But in case of GRB 190829A, it evolves as a hard to soft tracking trend for the first episode and then follows a very soft and disordered pattern for the second episode. The low energy spectral indices do not cross the synchrotron limits (from -1.5 to -0.67). But in the case of GRB 190114C and GRB 190829A, it crosses the synchrotron limits which could be due to the presence of some other component such as the black body in their prompt emission phase.

We modelled the afterglows of these GRBs using power-law or broken power-law models. We found that GRB 180720B had the highest decay of flux in optical bands. Some prompt flare activities were observed in GRB 180720B. A strong flare was detected in the X-ray and UV/optical light curve of GRB 190829A. In this GRB, early and late time Xray flux decay was found to be highest, after excluding the flaring emission from the light curve.

Finally, we conclude that the prompt and afterglow properties of these GRBs are very peculiar in nature. We found a few similarities in GRB 180720B and GRB 190114C. All of them were nearby GRBs and earlier nearby long GRBs observations suggest that at least some of them detected simultaneously with associated core-collapse supernovae (Pandey 2016). Interestingly, broad-line type 1c supernovae associated with all these events has been reported. The study of the associated supernova will give a deeper understanding of these $\mathrm{TeV}$ detected GRBs. A more detailed prompt and afterglow analysis of such bursts will be published in Gupta et al. (2020).

Acknowledgments: AA, SBP and RG acknowledge BRICS grant DST/IMRCD/BRICS/Pilotcall1/ProFCheap/201 7(G). This research has made use of data obtained through the HEASARC Online Service, provided by the NASA-GSFC, in support of NASA High Energy Astrophysics Program. This work is partially based in observations taken with the $10.4 \mathrm{~m}$ GTC located at the Spanish island of La Palma. This work has been partially supported by the Spanish Science Ministry "Centro de Excelencia Severo Ochoa" Program under grant SEV-2017-0709.

\section{REFERENCES}

Abdalla, H., Adam, R., Aharonian, F., et al. 2019, Natur, 575,464

Alexander, K. D., Laskar, T., Berger, E., et al. 2019, GCN, 23726, 1

Arnaud, K. A. 1996, ASPC, 101, 17 
Band, D. L. 1997, ApJ, 486, 928

Band, D., Matteson, J., Ford, L., et al. 1993, ApJ, 413, 281

Barthelmy, S. D., Cummings, J. R., Krimm, H. A., et al. 2018, GCN, 22998, 1

Bhat, P. N., Fishman, G. J., Meegan, C. A., et al. 1994, ApJ, 426, 604

Bissaldi, E., \& Racusin, J. L. 2018, GCN, 22980, 1

Castro-Tirado, A. J. 2008, LNEA, 3, 131 2011, AcPol, 51, 16

2016, 41st COSPAR Scientific Assembly, 41, 297, E1.18-10-16

Castro-Tirado, A. J., Hu, Y., Fernandez-Garcia, E., et al. 2019, GCN, 23708, 1

Chand, V., Pal, P. S., Banerjee, A., et al. 2019, arXiv e-prints, arXiv:1905.11844

Chand, V., Banerjee, A., Gupta, R., et al. 2020, arXiv e-prints, arXiv:2001.00648

Chandra, P., Nayana, A. J., Bhattacharya, D., et al. 2018, GCN, 23073, 1

Chandra, P., et al. 2019, GCN, 25627, 1

Cherry, M. L., Yoshida, A., Sakamoto, T., et al. 2018, GCN, 23042, 1

de Naurois, M., \& H. E. S. S. Collaboration 2019, GCN, 25566, 1

de Ugarte Postigo, A., Bremer, M., Kann, D. A., et al. 2019, GCN, 25589, 1

Dichiara, S., Bernardini, M. G., Burrows, D. N., et al. 2019, GCN, 25552, 1

Duan, M.-Y., \& Wang, X.-G. 2019, ApJ, 884, 61

Fraija, N., Dichiara, S., Pedreira, A. C. C. do E. S., et al. 2019, ApJ, 879, 26 2019, ApJ, 885, 29

Frederiks, D., Golenetskii, S., Aptekar, R., et al. 2018, GCN, 23011, 1 2019, GCN, 23737, 1

Goldstein, A., Veres, P., Burns, E., et al. 2017, ApJ, 848, 14

Golenetskii, S. V., Mazets, E. P., Aptekar, R. L., et al. 1983, Natur, 306, 451

Gropp, J. D., Kennea, J. A., Klingler, N. J., et al. 2019, GCN, 23688, 1

Gupta, R., et al., 2020, in preparation

Hamburg, R., Veres, P., Meegan, C., et al. 2019, GCN, 23707, 1

Joshi, J. C., \& Razzaque, S. 2019, arXiv e-prints, arXiv: 1911.01558

Kargatis, V. E., Liang, E. P., Hurley, K. C., et al. 1994, ApJ, 422, 260

Krimm, H. A., Barthelmy, S. D., Cummings, J. R., et al.
2019, GCN, 23724, 1

Kocevski, D., Omodei, N., Axelsson, M., et al. 2019, GCN, 23709, 1

Kumar, P. \& Zhang, B. 2015, PhR, 561, 1

Laros, J. G., Evans, W. D., Fenimore, E. E., et al. 1985, ApJ, 290, 728

Laskar, T., Alexander, K. D., Berger, E., et al. 2019, GCN, 23728, 1

Laskar, T., Bhandari, S., Schroeder, G., et al. 2019, GCN, 25676, 1

Lesage, S., Poolakkil, S., Fletcher, C., et al. 2019, GCN, 25575, 1

Lien, A. Y., Barthelmy, S. D., Cummings, J. R., et al. 2019, GCN, 25579, 1

Lipunov, V., Kornilov, V., Gorbovskoy, E., et al. 2012, ASInC, 7, 275

MAGIC Collaboration, Acciari, V. A., Ansoldi, S., et al. 2019, Natur, 575, 459

Mirzoyan, R., Noda, K., Moretti, E., et al. 2019, GCN, 23701, 1

Monageng, I., van der Horst, A. J., Woudt, P. A., et al. 2019, GCN, 25635, 1

Nava, L., Salvaterra, R., Ghirlanda, G., et al. 2012, MNRAS, 421, 1256

Negoro, H., Tanimoto, A., Nakajima, M., et al. 2018, GCN, 22993, 1

Pandey, S. B. 2016, RMxAC, 48, 83

Piran, T. 2004, RvMP, 76, 1143

Piron, F., Longo, F., Axelsson, M., et al. 2019, GCN, 25574, 1

Pittori, C., Ursi, A., Tavani, M., et al. 2019, GCN, 25577,1

Roberts, O. J., \& Meegan, C. 2018, GCN, 22981, 1

Ryde, F., \& Svensson, R. 1999, ApJ, 512, 693

Sfaradi, I., Bright, J., Horesh, A., et al. 2018, GCN, 23037, 1

Siegel, M. H., Burrows, D. N., Deich, A., et al. 2018, GCN, 22975, 1

Siegel, M. H., \& Gropp, J. D. 2019, GCN, 23725, 1

Tsvetkova, A., Golenetskii, S., Aptekar, R., et al. 2019, GCN, 25660, 1

Ursi, A., Tavani, M., Marisaldi, M., et al. 2019, GCN, 23712,1

Valeev, A. F., Castro-Tirado, A. J., Hu, Y.-D., et al. 2019, GCN, 25565, 1

Volvach, A. E., Volvach, L. N., \& Pozanenko, A. 2019, GCN, 23750, 1

Willingale, R., Starling, R. L. C., Beardmore, A. P., et al. 2013, MNRAS, 431, 394

Xiao, S., Li, C. K., Li, X. B., et al. 2019, GCN, 23716, 1 